\title{
Global Genomic Variations of Pathogenic Bacteria Salmonella Reading
}

\author{
Woo Hyung Lee1, Yein Jeon², Jonathan S. Chung,4, Woo Jun Shim ${ }^{4,5}$, Min Jae Shinn, \\ Seok Gyu Han ${ }^{4,6}$, Seunghan Baek ${ }^{6}$, James Kim ${ }^{7,8}$, Jaewoo Kim ${ }^{4,8}$, Paul S. Chung, \\ ${ }^{1}$ Biotechnology, School of Sciences, Hong Kong University of Science of Technology, Hong Kong, China \\ ${ }^{2}$ Fuzbien Technology Institute, Rockville, USA \\ ${ }^{3}$ Ernest Mario School of Pharmacy, Rutgers University, New Brunswick, USA \\ ${ }^{4}$ College of Letters \& Sciences, University of California, Los Angeles, USA \\ ${ }^{5}$ Biology, Bachelor of Science, California Institute of Technology, Pasadena, USA \\ ${ }^{6}$ School of Arts \& Sciences, Johns Hopkins University, Baltimore, USA \\ ${ }^{7}$ School of Arts \& Sciences, University of Michigan, Ann Arbor, USA \\ ${ }^{8}$ Youth with Talents, Fairfax, USA \\ Email: paulschung08@gmail.com
}

How to cite this paper: Lee, W.H., Jeon, Y., Chung, J.S., Shim, W.J., Shin, M.J., Han, S.G., Baek, S., Kim, J., Kim, J. and Chung, P.S. (2021) Global Genomic Variations of Pathogenic Bacteria Salmonella Reading. Computational Molecular Bioscience, 11, 57-68. https://doi.org/10.4236/cmb.2021.113004

Received: September 1, 2021

Accepted: September 27, 2021

Published: September 30, 2021

Copyright $\odot 2021$ by author(s) and Scientific Research Publishing Inc. This work is licensed under the Creative Commons Attribution International License (CC BY 4.0).

http://creativecommons.org/licenses/by/4.0/

(c) (i) Open Access

\begin{abstract}
US Centers for Disease Control and Prevention (CDC) estimates food-borne pathogenic Salmonella bacteria cause about 1.35 million infections, 26,500 hospitalizations, and 420 deaths in the U.S. every year. Outbreaks of multidrug-resistant Salmonella Reading linked mainly to raw turkey products and alfalfa sprouts have accounted for human illness including mortality. Therefore, we downloaded and compared genome sequences of 897 Salmonella Reading isolated from 27 resources in 11 countries at 21 year-points from the National Center for Biotechnology Information database. Isolates were clustered into 10 clades which consisted of 3 major and 7 minor or single node clades. Although 3 major clades included both Europe and American isolates, one clade consisted of 605 North American isolates out of 614 isolates. Evolutionary distance is more related to the continent than the source of isolation. The host source, continent (North America and Europe) and phylogenetic clade were related to the prevalence of isolates encoding Antimicrobial Resistance Genes (ARGs). Prevalence of prophages was greater in bovine and swine isolates than poultry and human isolates and the least prevalence was found in human isolates. Between continents, the prevalence of phage was greater in North American isolates than European. The diversity of virulence factors in swine isolates differed from poultry isolate while no difference was found among continents. In conclusion, evolutionary distance is related to isolation host source rather than the continent, and genome features were distinguished by host and cluster. Our genomic analysis implies that Salmonella Reading evolved inde-
\end{abstract}


pendently to environments within its lineages.

\section{Keywords}

Salmonella, Serovar Reading, Genome, Antimicrobial Resistance Genes, Virulence Factors, Phylogenomic Tree

\section{Introduction}

Salmonella, an important causative microorganism of food-borne diseases, has extensive attention for worldwide high incidence [1]. US Centers for Disease Control and Prevention (CDC) estimates food-borne pathogenic Salmonella bacteria cause about 1.35 million infections, 26,500 hospitalizations, and 420 deaths in the U.S. every year. Non-typhoidal Salmonella spp. is a leading cause of human food poisoning worldwide and is responsible for 93 million infections annually [2]. Outbreaks of Multidrug-Resistant (MDR) Salmonella Reading linked mainly to raw turkey products and Alfalfa sprouts have accounted for human illness including mortality. Since $S$. Reading was identified from a water supply in Reading, England in 1916, it has been reported from various sources including domestic animals, poultry, produce and environmental samples. Recently, two large outbreaks of $S$. Reading were reported. CDC [3] reported an outbreak from November 2017 through March 2019 and it was linked to living turkeys and raw turkey products, but no single source product or company was attributed to the entire outbreak. This outbreak resulted in 358 illnesses, 133 hospitalizations, and 1 death across 42 states. Public Health Agency of Canada (2020) also reported outbreaks of 130 cases of $S$. Reading linked with poultry in Canada.

MDR S. Newport, S. Typhimurium, and other Salmonella serovars and phage types have been associated with increased morbidity and mortality in humans and animals [4]. The emergence of bacteria ARGs occurs during the evolutionary process and vertical gene transfer [5]. The acquired resistance includes genetic exchanges through horizontal gene transfer with the acquisition of ARGs on mobile genetic elements [6]. The therapeutic and non-therapeutic uses of antibiotics in humans and animals have increased selective pressure [7]. Furthermore, pathogenic bacteria including Salmonella have been selected for ARGs due to the constant exposure to antibiotics [8].

Considering the recent multiple outbreaks in North America and its multidrug resistance, $S$. Reading might need to be better understood its ecology and evolution. This study aims to investigate the phylogenomic distance of and ARGs and genomic features in publicly available widespread $S$. Reading genomes. To achieve the purpose of this study, a comprehensive genomic investigation was performed to distinguish genomic changes related to host source and isolation locations. 


\section{Methods}

\subsection{Sequenced Isolates}

Sequenced $S$. enterica serovar Reading isolates from Africa (2 isolates), Asia (8), Europe (139), North America (731) and 17 isolates with no data for region were obtained from EnteroBase with associated metadata. This collection included isolates from various sources such as aquatic (3), bovine (44), canine (1), companion animal (3), environment (12), feed (11), food (8), human (91), laboratory (1), ovine (2), poultry (510), swine (87), wild animal (1) and no data (123).

\subsection{Serotype Prediction}

In Silico serotype prediction was performed with the Salmonella In Silico Typing Resource [9]. Only isolates with a predicted serotype of Reading were included in downstream analyses.

\subsection{Analysis of Genomes}

1) Core genome (cg) SNPs analysis: Parsnp was used to construct a non-redundant core genome single nucleotide polymorphisms (SNPs) of all 897 isolates and $S$. enterica serovar Typhi strain CT18 was used as reference for genome alignment.

2) Phylogeny reconstruction: Maximum Likelihood phylogenic tree was generated using MEGA [10]. Number of bootstrap replications, substitution model, rates of sites, gaps/missing data treatment, ML Heuristic method were 100, Tamura-Nei model, Gamma distributed with Invariant sites $(G+I)$ with 5 of Discrete Gamma Categories, partial deletion with $95 \%$ site coverage cutoff and Nearest-Neighbor-Interchange (NMI) with NJ/BioNJ initial tree, respectively.

3) Antimicrobial resistance genes: Resfinder 4.1 [11] on Center for Genomic Epidemiology was used to identify acquired antimicrobial resistance genes in each isolate. Resistance genes were classified to 22 groups of antimicrobial resistance such as Aminoglycoside, Beta-lactam, Colistin, Fluoroquinolone, Fosfomycin, Fusidic acid, Lincosamide, Macrolide, MLS (Macrolide, Lincosamide and Streptogramin B), metronidazole (5-nitroimidazole), Phenicol, Quinolone, Rifampicin, Streptogramin B, Sulphonamide, Tetracycline, Trimethoprim, Vancomycin, Vancomycin (Glycopeptid), co-resistance to Oxazolidinone and Phenicol, co-resistance to Fluoroquinolone and aminoglycoside, and co-resistance to Phenicols, Lincosamides, Oxazolidinones, Pleuromutilins, and Streptogramin A by the Resfinder.

4) Phage prevalence: A total of 23 Samonella phage assemblies in GenBank was downloaded through NCBI [12]. Phages were searched via BLASTn across all isolates. Less than 90 percent identities of matches were cutoff using a script.

5) Virulence Factors (VF) of pathogenic bacteria: Dataset containing 3663 VF-related non-redundant genes of which with curation was downloaded from Virulence factors of pathogenic bacteria website (http://www.mgc.ac.cn/VFs/). 


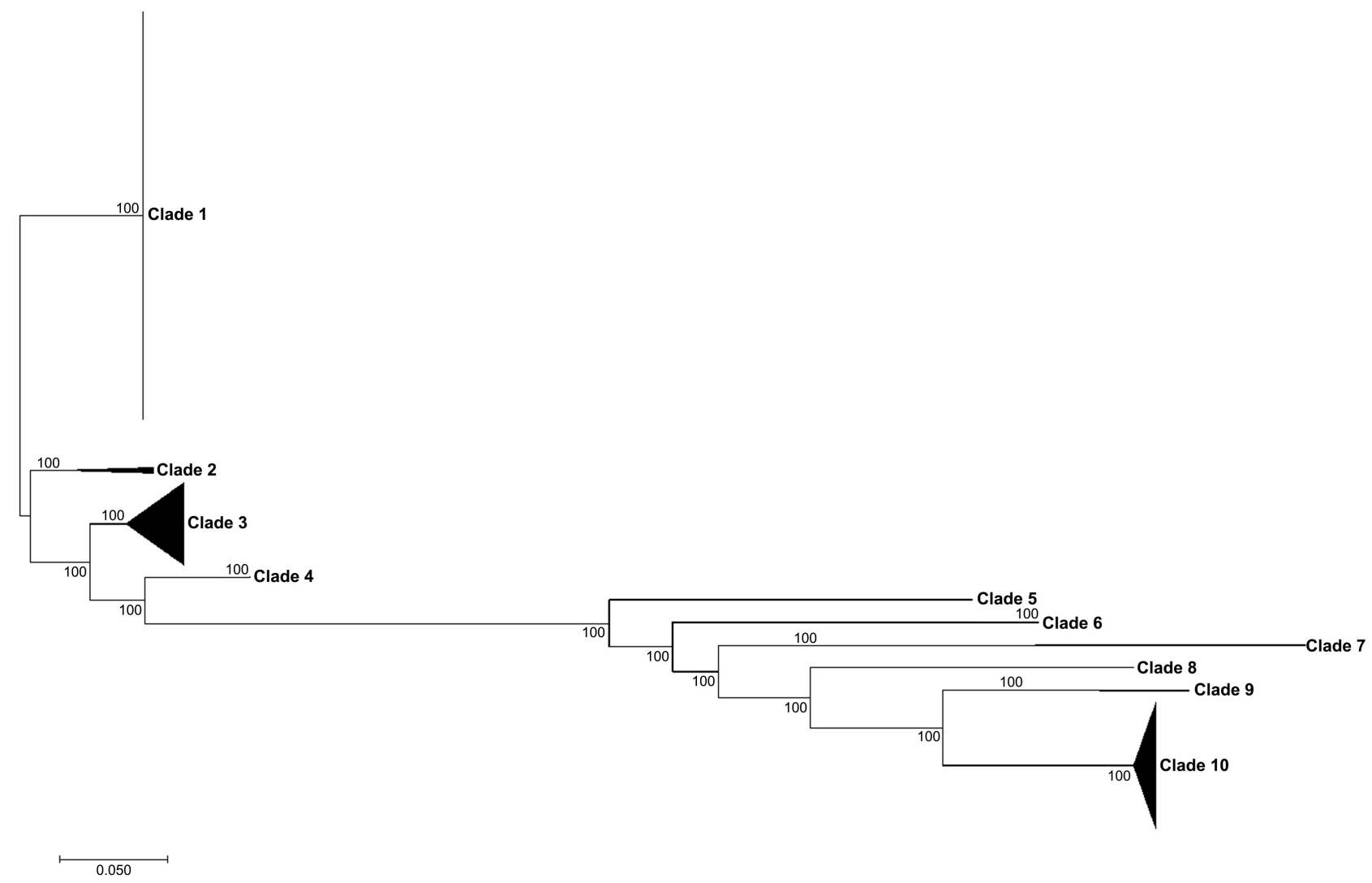

Figure 1. Maximum Likelihood phylogenetic tree of $S$. Reading isolates $(\mathrm{n}=897)$ based on 121,449 sites of core genome SNPs. Clades 1, 3 and 10 include 614, 105 and 160 isolates, respectively. Clades 2, 4, 5, 6, 7, 8 and 9 include 8, 2, 1, 2, 2, 1 and 2 isolates, respectively. Clade 1 consisted of 3 Europe and 605 North America including 497 poultry isolates. Clade 3 consisted of 67 Europe including 65 human isolates and 22 North America isolates. Clade 10 consisted of 60 Europe including 55 swine isolates and 97 North America isolates. 
ND. Clade 10 includes one Asian, 60 European, 97 North American and 2 ND isolates. For host sources, Clades 1 includes 497 poultry and few of bovine, canine, environment, feed, food, human, swine, wild animal and 90 of ND. Clade 3 includes 66 human isolates and few of aquatic, bovine, companion animal, environment, food, ovine, and 23 of ND. No poultry isolate was identified in Clade 3. Clade 10 includes 38 bovine, 11 poultry and 85 swine, and few of companion animal, environment, feed, and 8 ND. Poultry isolates dominate the Clade 1 and also identified in Clade 10 but not in Clade 3. Human isolates dominated in Clade 3 and also identified in Clades 1 and 10. Swine isolates dominated in Clade 10 and identified in Clade 1 but not in Clade 3. Two large outbreaks of S. Reading with raw turkey products in North America $(23,24)$ has been reported and in this study majority of poultry strains (497 isolates) are phylogenetically linked closely in Clade 1 but also two different clusters, Clades 6 and 10, were identified for North America poultry strains. Poultry stains clustered with swine stains in Clade 10 and are polyphyletic from stains in Clade 1 and at least two lineages existed for poultry stains. Among 3 major clusters, any distinct lineage was identified with continents, Europe and North America, and it might reflect the rapid dissemination of this pathogen worldwide through the increased human travels and the massive production and the broad distribution of food and feed resources.

Global emergence of pathogens in the same lineage may alert the spread of antimicrobial resistance genes and create new control challenges in food safety and public health on a global scale [13]. Although the ResFinder database included 22 groups of antimicrobial resistance, 7 resistance groups such as aminoglycoside, beta-lactamase, chloramphenicol, fosfomycin, quinolone, sulfonamide and tetracycline were mainly found in isolates. Prevalence of ARGs expressed as \% of isolates containing ARGs were shown in Figures 2-4. When all isolates were included, host effects $(\mathrm{p}<0.0001)$ on prevalence were observed for all ARGs (Figure 2(A)). Aminoglycoside, beta-lactamase and sulfonamide prevalence were greater in bovine and poultry than swine. Chloramphenicol prevalence was greater in bovine than in poultry and swine. Fosfomycin and tetracycline were greater in bovine and swine than poultry. Quinolone was greater in human than other hosts. Variation in AMR profile diversity both within and between food-production animal host species with higher AMR diversity of isolates from broiler compared to layer chickens, breeder compared to rearer and finisher pigs and beef compared to dairy cattle was reported and alternate sources of AMR bacteria and variation in selective evolutionary pressures within and between food-production animal host species populations was suggested [14].

Continent effects $(\mathrm{p}<0.0001)$ were also observed on all ARGs (Figure 2(B)). Aminoglycoside, beta-lactamase and sulfonamide prevalence were greater in North America isolates than Europe and chloramphenicol, fosfomycin, quinolone and tetracycline were greater in Europe than North America isolates. Considering the evolution and dissemination of pathogens in the same lineages, horizontal gene transfer might occur locally and under different pressures with local 


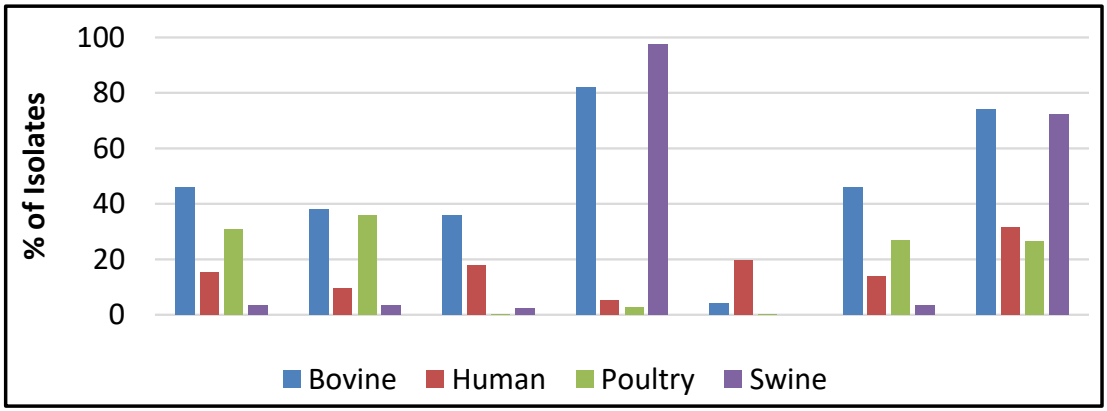

(A)

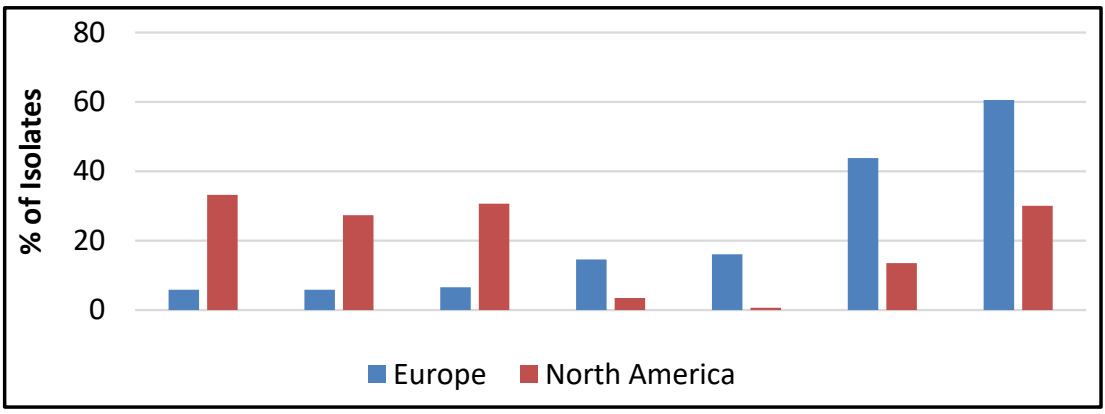

(B)

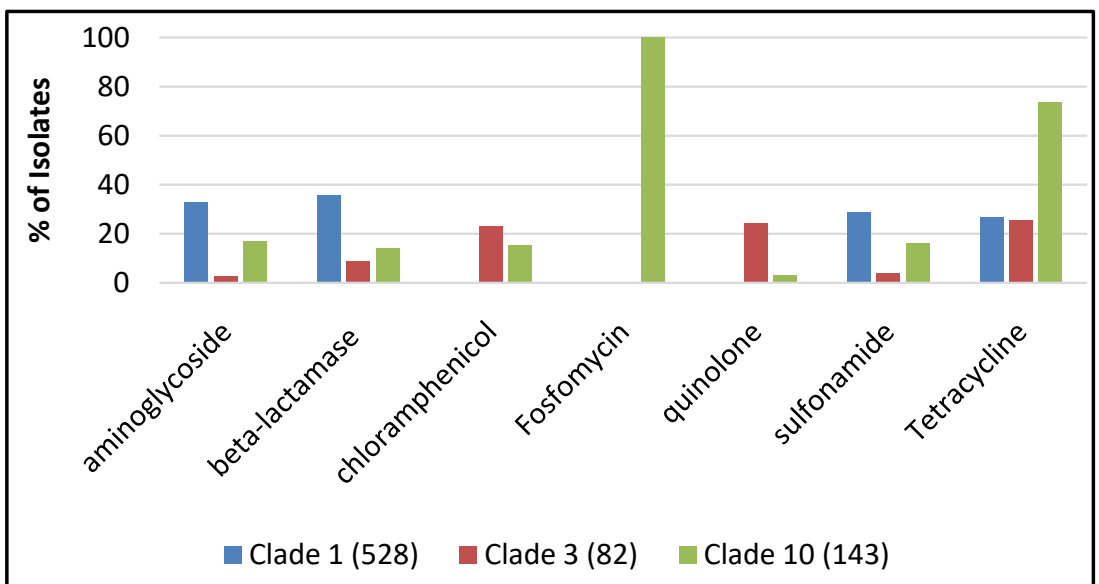

(C)

Figure 2. ARGs prevalence (\% of isolates containing ARGs) by host source (A), continents (B) and clades (C). ARGs groups in $\mathrm{C}$ also applied to A and B. (A) ARGs by Host Source; (B) ARGs by Continents; (C) ARGs by Clades.

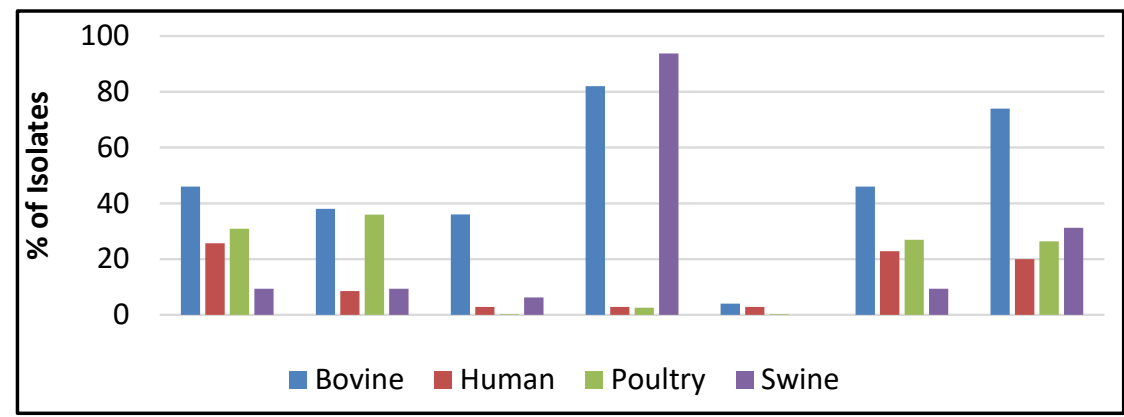

(A) 


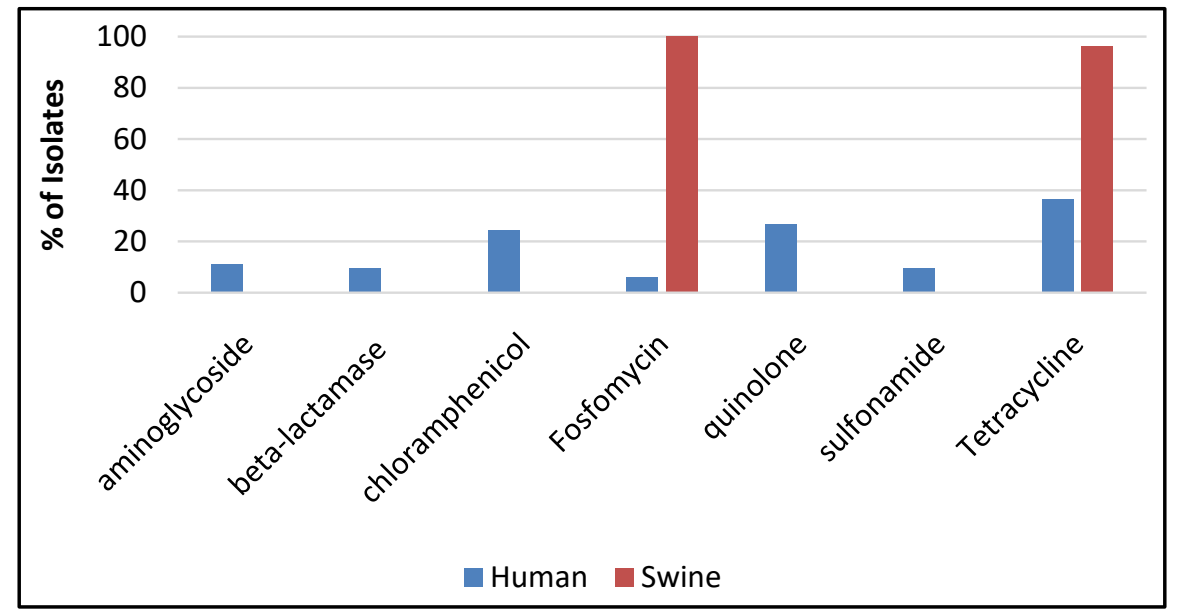

(B)

Figure 3. ARGs prevalence (\% of isolates containing ARGs) by host source in North American isolates (A) and European isolates (B). North America consisted of 43 bovine, 10 human, 510 Poultry and 32 swine isolates. Europe consisted of 82 human and 55 swine isolates. (A) ARGs in North American isolates; (B) ARGs in European isolates.

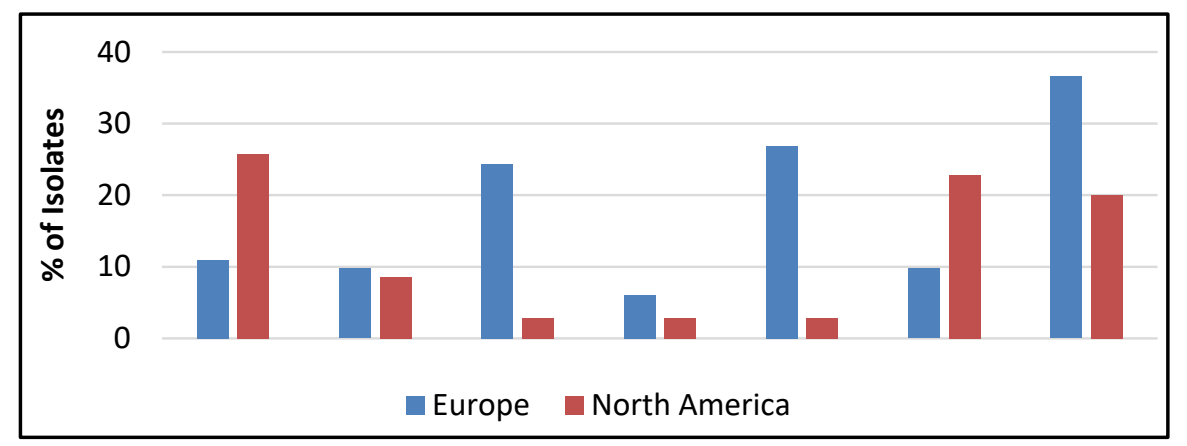

(A)

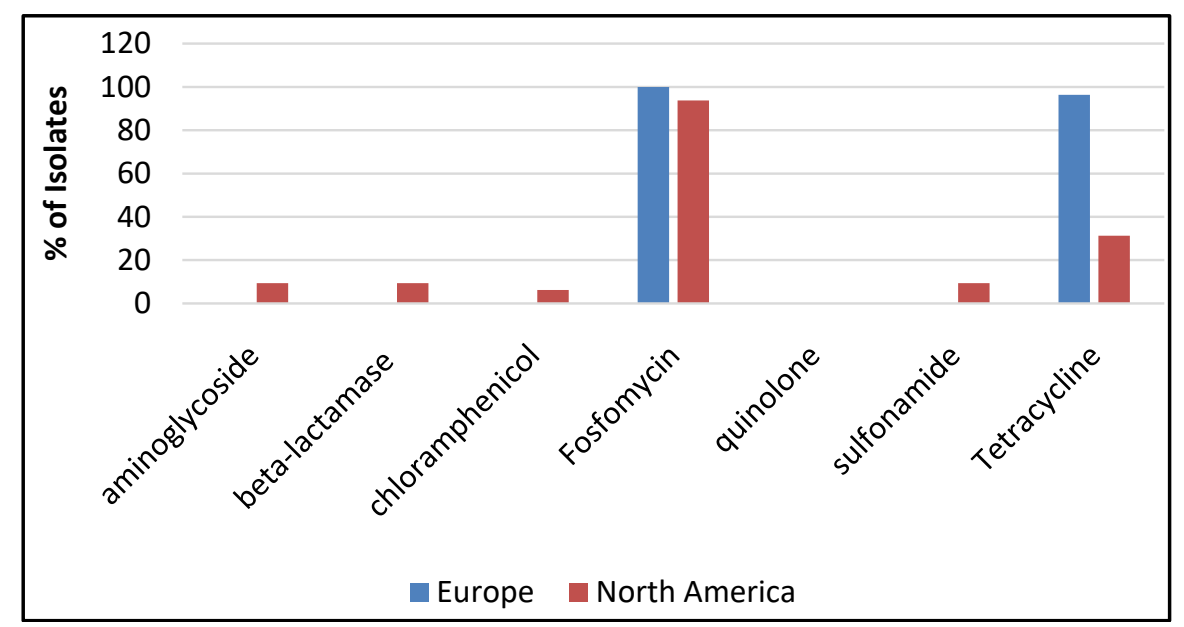

(B)

Figure 4. ARGs prevalence (\% of isolates containing ARGs) by continent in human (A) and Swine isolates (B). Europe consisted of 82 human, 55 swine, 1 environment and 1 ND isolates, and only human and swine isolates comparable between continents. (A) ARGs in human isolates; (B) ARGs in swine isolates. 
drug policies [15]. Clade 1 had greater $(\mathrm{p}<0.0001)$ prevalence of aminoglycoside, beta-lactamase, and sulfonamide than Clade 3 (Figure 2(C)). Clade 3 had greater prevalence of chloramphenicol and quinolone than Clade 1. Clade 10 contained higher prevalence of fosfomycin and tetracycline than Clades 1 and 3.

Host sources were compared in each continent to exclude the continent effect on host effects (Figure 3). ARGs prevalence is similar in North America isolates to in all isolates except chloramphenicol and tetracycline (Figure 3(A)). Chloramphenicol prevalence in bovine was greater $(\mathrm{p}<0.0001)$ than in human, and tetracycline prevalence were not significantly different between bovine and swine in North America isolates. In Europe isolates (Figure 3(B)), Human isolates had greater prevalence of aminoglycoside $(\mathrm{p}=0.0111)$, beta-lactamase $(\mathrm{p}=0.0214)$, chloramphenicol and quinolone $(\mathrm{p}<0.0001)$, and sulfonamide $(\mathrm{p}=0.0214)$ than swine, and swine had greater $(\mathrm{p}<0.0001)$ prevalence of fosfomycin and tetracycline than human isolates. For human isolates (Figure 4(A)), Europe isolates had greater prevalence of chloramphenicol $(\mathrm{p}=0.0038)$ and quinolone $(\mathrm{p}=$ 0.0019 , and for swine isolates (Figure 4(B)) prevalence of aminoglycoside, beta-lactamase, sulfonamide were greater $(\mathrm{p}=0.05)$ in North America isolates and the prevalence of tetracycline was greater $(p<0.0001)$ in Europe isolates.

Prevalence of bacteriophages (phages) was greater $(p<0.0001)$ in bovine and swine than poultry and human isolates (Figure 5). Poultry also had the greater $(\mathrm{p}<0.0001)$ prevalence of phage than human. Between continents, North America had greater $(\mathrm{p}=0.0061)$ prevalence of phage than Europe (Figure 5). Phages are viruses that target and destroy bacteria [16]. Research developing therapeutic phage use as antibiotics in animal industry is increasing due to their specificity, efficient bacterial lysis and their capability to self-replicate [17]. However, Salmonella phages appear to be abundant (up to 97\% prevalence) and widespread in animal feces, wastewater, and the environment of food animals [18] and the

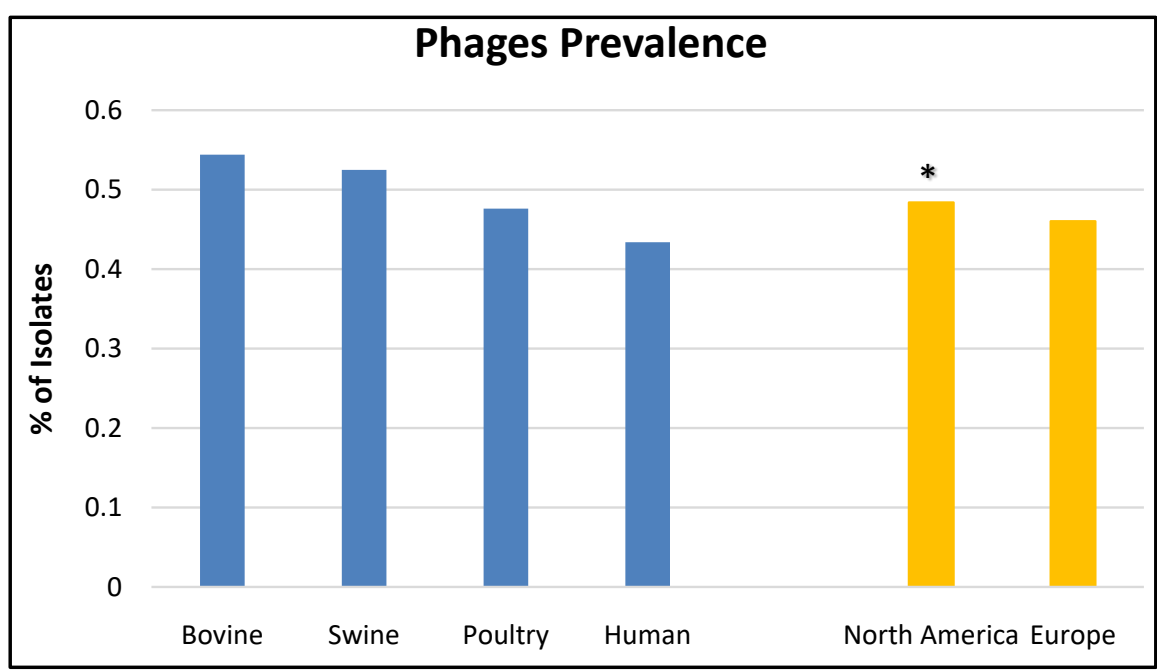

Figure 5. Phages prevalence (\% of isolates hovering phages) by host source (left, blue) or by continent (right, orange). ABCD Means with different scripts differ $(\mathrm{p}<0.0001)$. * Means with or without ${ }^{*}$ differ $(p=0.0061)$. 
diversity of phages are thought to have interactions between Salmonella serovars and specific phages [19]. Salmonella phages had different host range that US phages showed a narrow host range, whereas Thai phages showed a wide phage host range [20].

The diversity of virulence factors was different between Clades 1 and 3 vs Clade 10. Europe strains are more $(\mathrm{p}<0.0001)$ than North America stains in Clade 3 while more North America strains than Europe strains are in Clades 1 and 10 , thus the difference in virulence profile was not caused by the continent source. Virulence factor diversity was also different between poultry and swine isolates. Between Europe and North America, no difference in virulence factor diversity was found (data not shown). Virulence factors enable the bacterial adhesion, invasion and all intracellular survival of Salmonella. Many virulence factors are encoded on Salmonella Pathogenicity Islands (SPI) on the chromosome [21]. The most of SPI is conserved in serovars and the different profile of virulence factors in $S$. Reading implies the loss or acquirement of genes to adapt to host sources and maintained in their lineage but not within the continent.

In conclusion, evolutionary distance is related to isolation host source rather than continent, and genome features were distinguished by host and cluster. Our genomic analysis implies that Salmonella Reading evolved independently to environments within its lineages.

\section{Summary}

Two large outbreaks of $S$. Reading with raw turkey products in North America have been reported, the study found that the majority of poultry strains are phylogenetically linked in multi-clades identified for North American poultry strains. Poultry stains clustered with swine stains and are polyphyletic and at least two lineages existed for poultry stains.

Aminoglycoside, beta-lactamase, chloramphenicol, fosfomycin, quinolone, sulfonamide and tetracycline ARGs were found in isolates. Bovine and poultry showed greater prevalence in aminoglycoside, beta-lactam and sulfonamide resistances than in swine. Chloramphenicol prevalence was greater in bovine than poultry and swine. Fosfomycin and tetracycline prevalences were greater in bovine and swine than poultry while quinolone was greater in humans than in any other hosts. Aminoglycoside, beta-lactam and sulfonamide prevalences were greater in North America than in Europe. Chloramphenicol, fosfomycin, quinolone and tetracycline were greater in Europe than in North America. Considering the evolution and dissemination of pathogens in the same lineages, horizontal gene transfer might occur locally under varying pressures associated with local drug policies.

Prevalence of bacteriophages in bovine and swine were greater than poultry and human. Poultry also had a greater prevalence of phage than humans. North America displayed a greater prevalence of phage than Europe. Europe strains showed higher diversity of virulence factors than North America stains in Clade 
3 while higher diversity of VF was observed in North America strains for Clades 1 and 10, clarifying that the difference in VF was not caused by the continent source. VF diversity also differed in poultry and swine isolates. Observation showing that most of SPI conserved in serotypes and different profiles of virulence factors in $S$. Reading imply the loss or acquirement of genes to adapt to host sources and maintained in their lineage but not within the continent.

From the study, the rapid worldwide dissemination of pathogens including $S$. Reading can be attributed to increased human travel and large-scale and broad distribution of food and feed resources.

\section{Acknowledgements}

The authors acknowledge the Fuzbien Technology Institute and Youth with Talents for sponsoring this research project (FTI-7-2020).

\section{Conflicts of Interest}

The authors declare no conflicts of interest regarding the publication of this paper.

\section{References}

[1] Muvhali, M., Smith, A.M., Rakgantso, A.M. and Keddy, K.H. (2017) Investigation of Salmonella Enteritidis Outbreaks in South Africa Using Multi-Locus Variable-Number Tandem-Repeats Analysis, 2013-2015. BMC Infectious Diseases, 17, Article No. 661. https://doi.org/10.1186/s12879-017-2751-8

[2] Torgerson, P.R., Devleesschauwer, B., Praet, N., Speybroeck, N., Willingham, A.L., Kasuga, F., et al. (2015) World Health Organization Estimates of the Global and Regional Disease Burden of 11 Foodborne Parasitic Diseases, 2010: A Data Synthesis. PLoS Medicine, 12, Article ID: e1001920. https://doi.org/10.1371/journal.pmed.1001920

[3] Centers for Disease Control and Prevention (2019) Outbreak of Multidrug-Resistant Salmonella Infections Linked to Raw Turkey Products: Final Update. Centers for Disease Control and Prevention, Atlanta. https://www.cdc.gov/salmonella/reading-07-18/index.html

[4] Gupta, A., Fontana, J., Crowe, C., Bolstorff, B., Stout, A., Van Duyne, S., Hoekstra, M.P., Whichard, J.M., Barrett, T.J., Angulo, F.J. and National Antimicrobial Resistance Monitoring System PulseNet Working Group (2003) Emergence of Multidrug-Resistant Salmonella enterica Serotype Newport Infections Resistant to Expanded-Spectrum Cephalosporins in the United States. Journal of Infectious Diseases, 188, 1707-1716. https://doi.org/10.1086/379668

[5] Founou, L.L., Founou, R.C. and Essack, S.Y. (2016) Antibiotic Resistance in the Food Chain: A Developing Country-Perspective. Frontiers in Microbiology, 7, Article No. 1881. https://doi.org/10.3389/fmicb.2016.01881

[6] Kudirkiene, E., Andoh, L.A., Ahmed, S., Herrero-Fresno, A., Dalsgaard, A., Obiri-Danso, K., et al. (2018) The Use of a Combined Bioinformatics Approach to Locate Antibiotic Resistance Genes on Plasmids from Whole Genome Sequences of Salmonella enterica Serovars from Humans in Ghana. Frontiers in Microbiology, 9, Article No. 1010. https://doi.org/10.3389/fmicb.2018.01010

[7] O’Neill, J. (2016) Tackling Drug-Resistant Infections Globally: Final Report and Rec- 
ommendations. Review on Antimicrobial Resistance, Government of the United Kingdom, 1-84.

[8] Rabello, R.F., Bonelli, R.R., Penna, B.A., Albuquerque, J.P., Souza, R.M. and Cerqueira, A.M.F. (2020) Antimicrobial Resistance in Farm Animals in Brazil: An Update Overview. Animals, 10, Article No. 552. https://doi.org/10.3390/ani10040552

[9] Yoshida, C., Kruczkiewicz, P., Laing, C.R., Lingohr, E.J., Gannon, V.P.J., Nash, J.H.E. and Taboada, E.N. (2016) The Salmonella in Silico Typing Resource (SISTR): An Open Web-Accessible Tool for Rapidly Typing and Subtyping Draft Salmonella Genome Assemblies. PLoS ONE, 11, Article ID: e0147101. https://doi.org/10.1371/journal.pone.0147101

[10] Kumar, S., Nei, M., Dudley, J. and Tamura, K. (2008) MEGA: A Biologist-Centric Software for Evolutionary Analysis of DNA and Protein Sequences. Briefings in Bioinformatics, 9, 299-306. https://doi.org/10.1093/bib/bbn017

[11] Bortolaia, V., Kaas, R.F., Ruppe, E., Roberts, M.C., Schwarz, S., Cattoir, V., Philippon, A., Allesoe, R.L., Rebelo, A.R., Florensa, A.R., Fagelhauer, L., Chakraborty, T., Neumann, B., Werner, G., Bender, J.K., Stingl, K., Nguyen, M., Coppens, J., Xavier, B.B., Malhotra-Kumar, S., Westh, H., Pinholt, M., Anjum, M.F., Duggett, N.A., Kempf, I., Nykäsenoja, S., Olkkola, S., Wieczorek, K., Amaro, A., Clemente, L., Mossong, J., Losch, S., Ragimbeau, C., Lund, O. and Aarestrup, F.M. (2020) ResFinder 4.0 for Predictions of Phenotypes from Genotypes. Journal of Antimicrobial Chemotherapy, 75, 3491-3500. https://doi.org/10.1093/jac/dkaa345

[12] NCBI (National Centre for Biotechnology Information) (1988) Basic Local Alignment Search Tools. National Centre for Biotechnology Information, Rockville.

[13] Majowicz, S.E., Musto, J., Scallan, E., Angulo, F.J., Kirk, M., O’Brien, S.J., et al. (2010) The Global Burden of Nontyphoidal Salmonella Gastroenteritis. Clinical Infectious Diseases, 50, 882-889. https://doi.org/10.1086/650733

[14] Mellor, K.C., Petrovska, L., Thomson, N.R., Harris, K., Reid, S.W.J. and Mather, A.E. (2019) Antimicrobial Resistance Diversity Suggestive of Distinct Salmonella Typhimurium Sources or Selective Pressures in Food-Production Animals. Frontiers in Microbiology, 10, Article No. 708. https://doi.org/10.3389/fmicb.2019.00708

[15] Davis, M.A., Hancock, D.D. and Besser, T.E. (2002) Multiresistant Clones of Salmonella enterica: The Importance of Dissemination. Journal of Laboratory and Clinical Medicine, 140, 135-141. https://doi.org/10.1067/mlc.2002.126411

[16] Borie, C., Robeson, J. and Galarce, N. (2014) Lytic Bacteriophages in Veterinary Medicine: A Therapeutic Option against Bacterial Pathogens. Archivos de Medicina Veterinaria, 46, 167-179. https://doi.org/10.4067/S0301-732X2014000200002

[17] Nobrega, F.L., Costa, A.R., Kluskens, L.D. and Azeredo, J. (2015) Revisiting Phage Therapy: New Applications for Old Resources. Trends in Microbiology, 23, 185-191. https://doi.org/10.1016/j.tim.2015.01.006

[18] Pulido, R.P., Burgos, M.J.G., Gálvez, A. and López, R.L. (2016) Application of Bacteriophages in Post-Harvest Control of Human Pathogenic and Food Spoiling Bacteria. Critical Reviews in Biotechnology, 36, 851-861. https://doi.org/10.3109/07388551.2015.1049935

[19] Switt, A.I.M., den Bakker, H.C., Vongkamjan, K., Hoelzer, K., Warnick. L.D., Cummings, K.J. and Wiedmann, M. (2013) Salmonella Bacteriophage Diversity Reflects Host Diversity on Dairy Farms. Food Microbiology, 36, 275-285. https://doi.org/10.1016/j.fm.2013.06.014

[20] Wongsuntornpoj, S., Switt, A.I.M., Bergholz, P., Wiedmann, M. and Chaturongakul, S. (2014) Salmonella Phages Isolated from Dairy Farms in Thailand Show Wid- 
er Host Range than a Comparable Set of Phages Isolated from U.S. Dairy Farms. Veterinary Microbiology, 172, 345-352.

https://doi.org/10.1016/j.vetmic.2014.05.023

[21] Que, F., Wu, S. and Huang, R. (2013) Salmonella Pathogenicity Island 1(SPI-1) at Work. Current Microbiology, 66, 582-587.

https://doi.org/10.1007/s00284-013-0307-8 